\title{
DOR MUSCULAR EM CABEÇA E PESCOÇO E MEDIDAS VOCAIS ACÚSTICAS DE FONTE GLÓTICA
}

\author{
Head and neck muscles pain and glottic \\ source acoustical vocal Measures
}

\author{
Luane de Moraes Boton (1), Marcela Forgiarini Morisso (2), \\ Ana Maria Toniolo da Silva ${ }^{(3)}$, Carla Aparecida Cielo ${ }^{(4)}$
}

\begin{abstract}
RESUMO
Objetivo: verificar a relação entre a presença de dor na musculatura da cabeça, face, boca e pescoço envolvidos no processo fisiológico da função mastigatória e nos aspectos lateral e posterior da articulação temporomandibular (ATM) e medidas vocais acústicas da fonte glótica. Método: amostra de 24 mulheres com idades entre 16 e 56 anos com queixas e sintomatologia de Disfunção Temporomandibular (DTM). Aplicação de um questionário de anamnese, exame clínico específico para verificação da presença de dor na musculatura da cabeça, face, boca e pescoço e nas ATM; avaliações otorrinolaringológica, do sistema estomatognático, audiológica e gravação digital da voz com análise vocal acústica da fonte glótica por meio do software Multi Dimensional Voice Program Advanced da KayPENTAX. Os resultados obtidos foram analisados estatisticamente pelo teste do qui-quadrado ao nível de significância de 0,05. Resultados: houve significância estatística entre: ausência de dor no masseter superficial e alteração do índice de turbulência vocal (VTI); ausência de dor no aspecto posterior da ATM e alteração do coeficiente de perturbação do pitch (PPQ) e alteração da variação da frequência fundamental (vfo); presença de dor no pterigóideo medial e normalidade do grau de quebra vocal (DVB). Conclusão: verificou-se que não houve relação entre as medidas de voz alteradas e a presença de dor nos músculos avaliados, mas algumas medidas alteradas se relacionaram com a ausência de dor muscular, sugerindo que outros aspectos da DTM, que não a dor, podem ocasionar alteração de medidas vocais acústicas.
\end{abstract}

DESCRITORES: Articulação Temporomandibular; Dor Facial; Distúrbios da Voz

(1) Fonoaudióloga; INTERSAÚDE- Cooperativa dos profissionais autônomos da área da saúde; Mestranda em Distúrbios da Comunicação Humana na Universidade Federal de Santa Maria, UFSM, Santa Maria, RS.

(2) Fonoaudióloga; Associação dos Pais e Amigos dos Excepcionais - APAE, Candelária, Rio Grande do Sul; Mestre em Distúrbios da Comunicação Humana pela Universidade Federal de Santa Maria, UFSM, Santa Maria, RS.

(3) Fonoaudióloga; Professora Associada do Departamento de Fonoaudiologia da Universidade Federal de Santa Maria, UFSM, Santa Maria, RS; Doutora em Ciências dos Distúrbios da Comunicação Humana pela Universidade Federal de São Paulo.

(4) Fonoaudióloga; Professora Adjunta do Departamento de Fonoaudiologia e do Programa de Pós-Graduação em Distúrbios da Comunicação Humana da Universidade Federal de Santa Maria, UFSM, Santa Maria, RS; Doutora em Linguística Aplicada pela Pontifícia Universidade Católica do Rio Grande do Sul.

Conflito de interesses: inexistente

\section{INTRODUÇÃO}

A articulação temporomandibular (ATM) tem sido frequentemente estudada no campo da Fonoaudiologia por ser responsável pelos movimentos mandibulares e, consequentemente, pela eficiência das funções estomatognáticas que propiciam clareza na fala e na voz. Neste sentido, o estudo da ATM e de seus distúrbios passa a ser fundamental para a área fonoaudiológica.

A disfunção temporomandibular (DTM) constitui uma condição na qual há desarmonia no sistema estomatognático, podendo ocorrer envolvimento e prejuízo nos músculos mastigatórios, na ATM propriamente dita, ou em ambos ${ }^{1}$.

Desta forma, qualquer alteração na inter-relação de funções e estruturas pode vir a causar distúrbios 
fonoaudiológicos ${ }^{2}$ como prejuízos na articulação da fala e na qualidade da voz ${ }^{3}$. Além disso, desequilíbrios musculares durante a produção da voz podem gerar o que é conhecido como disfonia 4 .

A DTM limita ou impede a movimentação da mandíbula ${ }^{5}$, levando ao decréscimo da efetividade na produção vocal, pois o sujeito com DTM pode desenvolver ajustes impróprios da musculatura supra-hioidea, a fim de suprir tais deficiências e obter, consequentemente, uma voz alterada ${ }^{6}$.

Deste modo, a DTM e a dor orofacial são problemas que atingem grande parte da população e têm como sinais clínicos a limitação dos movimentos mandibulares ${ }^{7}$ que, consequentemente, também podem interferir na acústica da $\mathrm{voz}^{3}$.

No entanto, poucos são os relatos em relação à frequência e ao grau de comprometimento desses movimentos e, por extensão, da ATM envolvida nas alterações musculares relacionadas à voz ${ }^{8}$

As DTM constituem um termo que abrange uma série de problemas clínicos envolvendo, além da musculatura da cabeça, face, boca e pescoço, outras estruturas associadas. É importante mencionar que os trabalhos não relacionam a presença de queixas e sintomatologias de DTM com o padrão vocal destes sujeitos, nem mesmo relatam a presença de queixas vocais como sinais de DTM.

Sinais e sintomas de DTM são caracterizados por dor dos músculos da cabeça, pescoço e mastigatórios, dor em uma ou ambas ATM, movimentos limitados da mandíbula, ruídos articulares, deformidades faciais e cefaléias e ainda o hábito de ranger os dentes que é freqüentemente presente nesses quadros $^{9-11}$.

Quanto aos aspectos relacionados à etiologia, considera-se que a DTM é multifatorial, associada a fatores predisponentes iniciadores e perpetuantes $^{12}$ que incluem distúrbios da oclusão, das bases ósseas maxilar e mandibular, fatores traumáticos, problemas degenerativos, alterações musculares como hiperatividade ou hipoatividade, modificações funcionais e hábitos orais deletérios que podem levar à sobrecarga persistente na ATM ou na musculatura ${ }^{13}$. Além disso, podem ser considerados fatores causadores o estresse e problemas emocionais ${ }^{2}$.

Em vista da possível relação existente entre a qualidade vocal e a dor na musculatura da cabeça, face, boca e pescoço e nos aspectos lateral e posterior da ATM é que se justifica a importância da realização deste trabalho.

Assim, o presente estudo teve por objetivo verificar a relação entre a presença de dor na musculatura da cabeça, face, boca e pescoço envolvidos no processo fisiológico da função mastigatória e nos aspectos lateral e posterior da ATM e medidas vocais acústicas da fonte glótica em mulheres com queixas e sintomatologia de DTM.

\section{MÉTODO}

Esta pesquisa se caracterizou por ser um estudo transversal, quantitativo, retrospectivo com estudo em banco de dados. Foi realizada com indivíduos que procuraram o Departamento de Odontologia de duas instituições de ensino superior que foram contatados para uma investigação clínica.

Foram considerados como critérios de inclusão no estudo:

- apresentarem diagnóstico de queixas e sintomatologia de DTM;

- serem adultos do sexo feminino;

- aderirem ao Termo de Consentimento Livre e Esclarecido (TCLE).

Foram considerados critérios de exclusão:

- história pregressa ou presença de disfonia funcional ou organofuncional;

- história de tratamento odontológico, fisioterápico, medicamentoso e/ou fonoaudiológico prévio ou durante a coleta dos dados;

- relato de problemas neurológicos, hormonais, digestivos, faríngeos, laríngeos e/ou pulmonares, doenças metabólicas como diabetes ou hipertireoidismo

- problemas auditivos de ordem exógena ou endógena;

- diagnóstico de insuficiência ou incompetência velofaríngea, respiração predominantemente oral, alterações laríngeas ou outras alterações do sistema estomatognático.

Para a seleção da amostra, foram aplicados os critérios de inclusão e de exclusão por meio da utilização do Termo de Consentimento Livre e Esclarecido (TCLE); de um questionário de anamnese composto de dez perguntas referentes às principais queixas e sintomas de DTM, como a constatação da presença de dor, hábitos parafuncionais, limitação de movimentos mandibulares, ruídos articulares, percepção subjetiva de maloclusão e tensão emocional. Para cada pergunta, somente uma resposta deveria ser escolhida. Para cada resposta, foram atribuídos valores: Sim=10; Não=zero e Às vezes $=5$. Resultados de $0-15$ foram considerados como uma classificação provisória de não portador de DTM e os valores acima de 15 foram classificados como sintomatologia de DTM ${ }^{14}$.

Também foram colhidas informações sobre dados pessoais, histórico de saúde, queixas de voz, histórico de tratamentos. Ainda, foram realizadas avaliação otorrinolaringológica, para verificar as condições laríngeas, velofaríngeas e 
respiratórias, e fonoaudiológica para verificar estruturas e funções do sistema estomatognático, além de avaliação audiológica. Todos os sujeitos com alterações nessas avaliações foram excluídos e encaminhados para tratamento.

Desta forma, a amostra foi composta por 24 indivíduos adultos do sexo feminino, com idades variando entre 16 e 56 anos, que apresentavam queixa e sintomatologia de DTM.

Para a coleta de dados, os indivíduos passaram pelas seguintes avaliações:

- avaliação odontológica: a palpação muscular digital é o método mais aceito para determinar a dor muscular de um músculo específico cujo tecido foi comprometido por trauma ou fadiga ${ }^{15}$. Este exame avaliou grupos musculares da cabeça, face, boca e pescoço envolvidos no processo fisiológico da função mastigatória; os músculos externos da face-músculos temporal, nos três feixes, anterior, médio e posterior (D) e (E), pterigóideo medial (D) e (E), masséter superficial (D) e (E), trapézio (D) e (E), e músculo esternocleido-mastóideo (D) e (E); os músculos da boca- músculo masséter, feixe profundo (D) e $(E)$, inserção do tendão do músculo temporal no espaço retromolar (D) e (E); e, ainda, os músculos cervicais posteriores. Para a avaliação das ATM, foi realizada a palpação dos seus aspectos lateral e posterior. Dessa forma, foi efetuada, pelo dentista uma única pressão firme, com duração de 1 a 2 segundos sobre os principais músculos da mastigação e sobre os músculos que fornecem apoio secundário, como os do pescoço. Durante a palpação, perguntouse ao paciente se sentia dor ou apenas desconforto. Somente na avaliação do músculo masseter, feixe superficial, foi solicitado que o participante realizasse contração isométrica por meio de apertamento dental.

$\mathrm{Na}$ avaliação dental, foram observadas: presença de desgaste dental; ausência de dentes; oclusão ${ }^{16}$; função oclusal de guia em canino; função oclusal de guia anterior e função oclusal em grupo.

- avaliação fonoaudiológica: realizada por fonoaudiólogo por meio de análise acústica da emissão sustentada da vogal [a:], em tom e intensidade habituais, até o final da expiração, colhida com o sujeito em pé, gravador digital Panasonic RR-US380, microfone a $4 \mathrm{~cm}$ da boca do sujeito. O início e o final da emissão foram descartados para evitar a interferência do ataque vocal e do decréscimo de intensidade do final da emissão na análise. Para a extração de medidas vocais acústicas, foi adotado o Multi Dimensional Voice Program Advanced, Model 5105, versão 2.30, da Kay PENTAX, que fornece dados sobre o sinal da fonte glótica, apresentando também os limiares de normalidade. Foram obtidos os valores de frequência fundamental (f0), frequência fundamental média (Mf0), quociente de perturbação do pitch (PPQ), coeficiente de variação da frequência fundamental (vf0), quociente de perturbação da amplitude (APQ), proporção harmônico-ruído (NHR), índice de turbulência da voz (VTI), índice de fonação suave (SPI), grau de quebra de voz (DVB) e grau de componentes sub-harmônicos (DSH).

Este trabalho foi previamente aprovado pelo Comitê de Ética em Pesquisa da Instituição, sob o protocolo de número 116. Todos os indivíduos receberam informações sobre a pesquisa e assinaram o TCLE, conforme a resolução 196/96 da Comissão Nacional de Ética em Pesquisa.

Após as avaliações, os dados encontrados foram tratados por meio de análise estatística. O teste do Qui-Quadrado para variável de independência foi realizado para o cruzamento entre a presença de dor nos grupos musculares da cabeça, face, boca e pescoço e nos aspectos lateral e posterior da ATM com as medidas acústicas da voz, sendo considerado o nível de significância de $5 \%(p<0,05)$.

\section{RESULTADOS}

Os resultados da comparação entre as medidas acústicas da fonte glótica e a palpação muscular digital são apresentados nas Tabelas 1, 2, 3 e 4. 


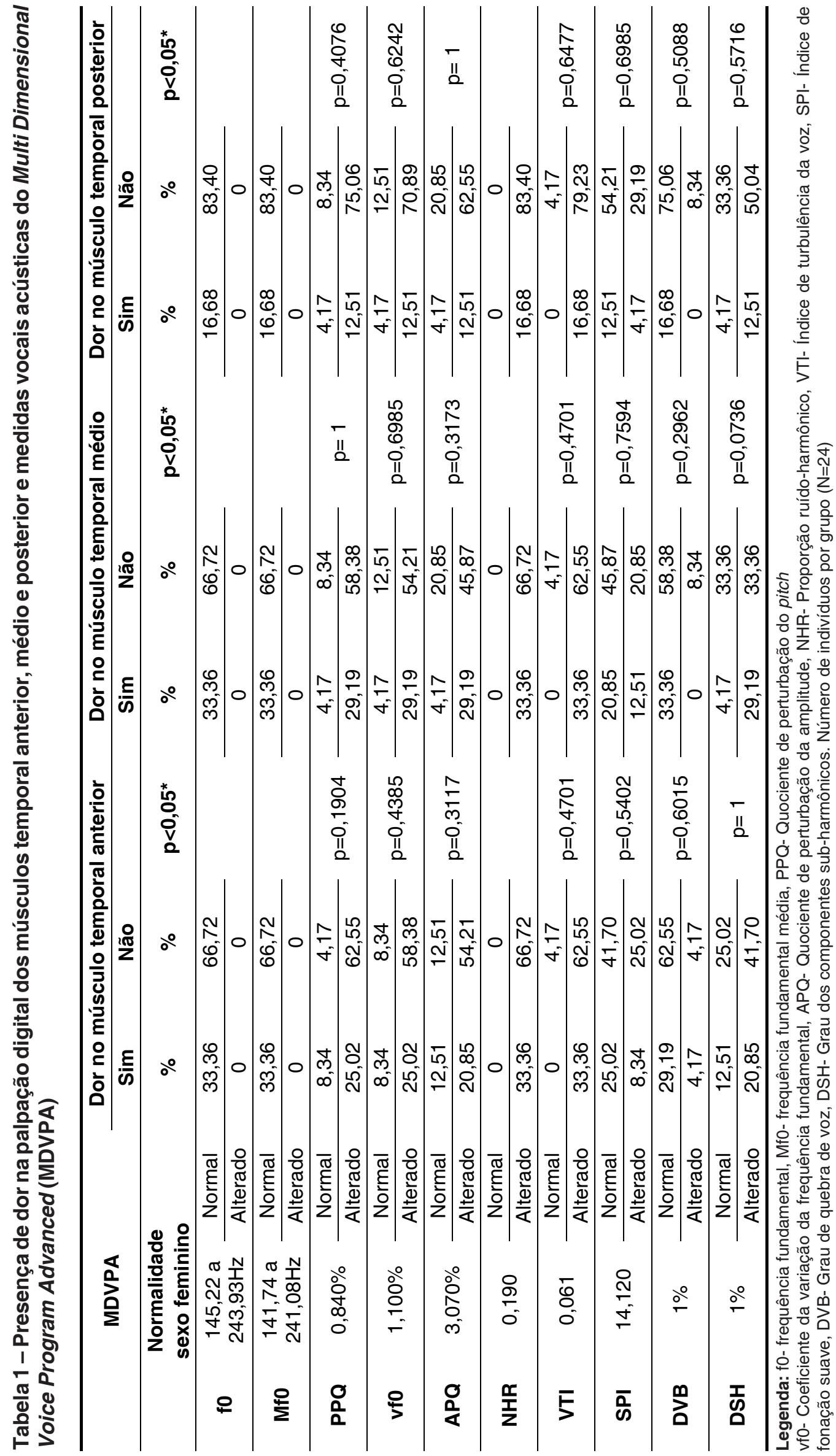




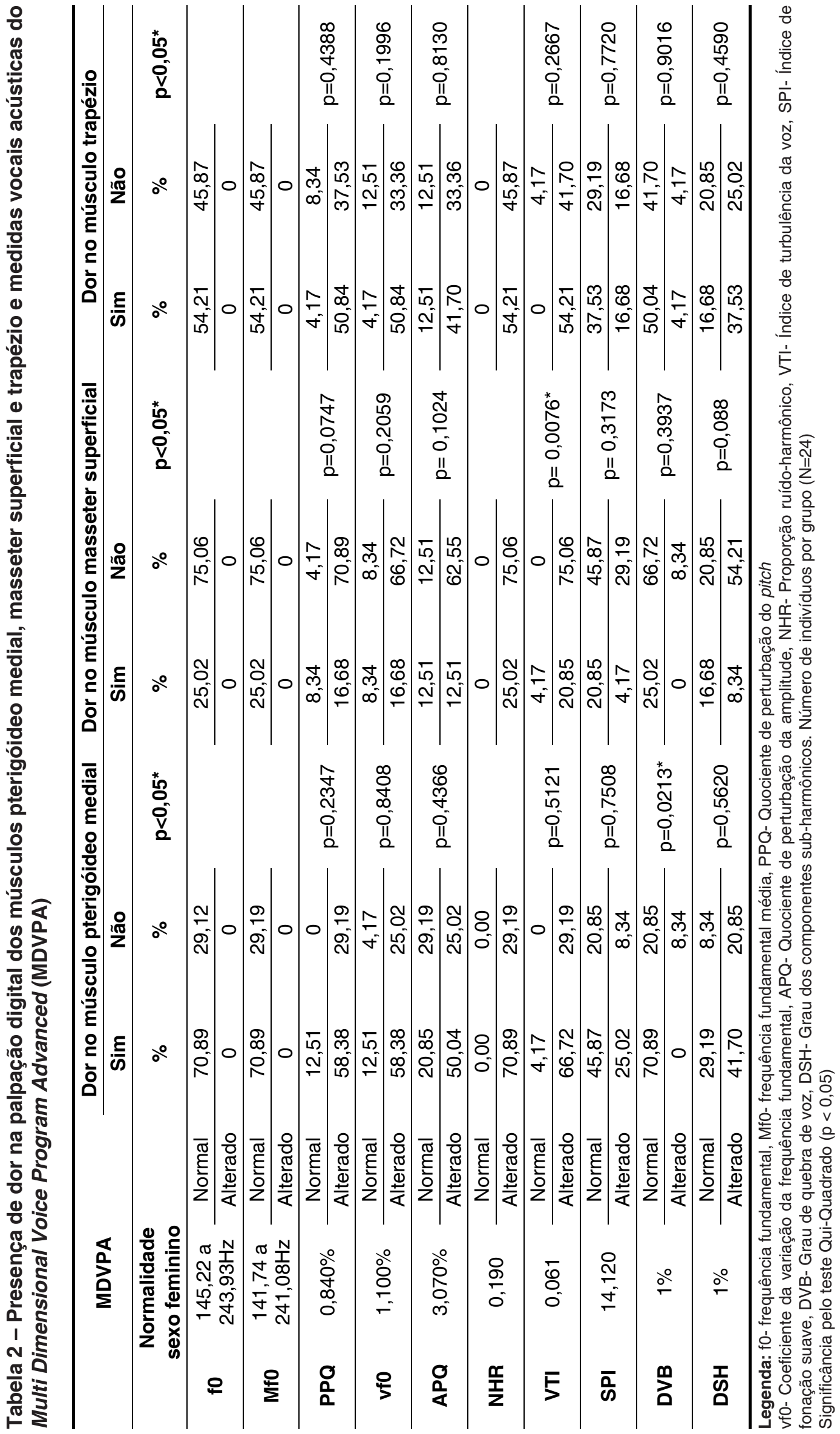




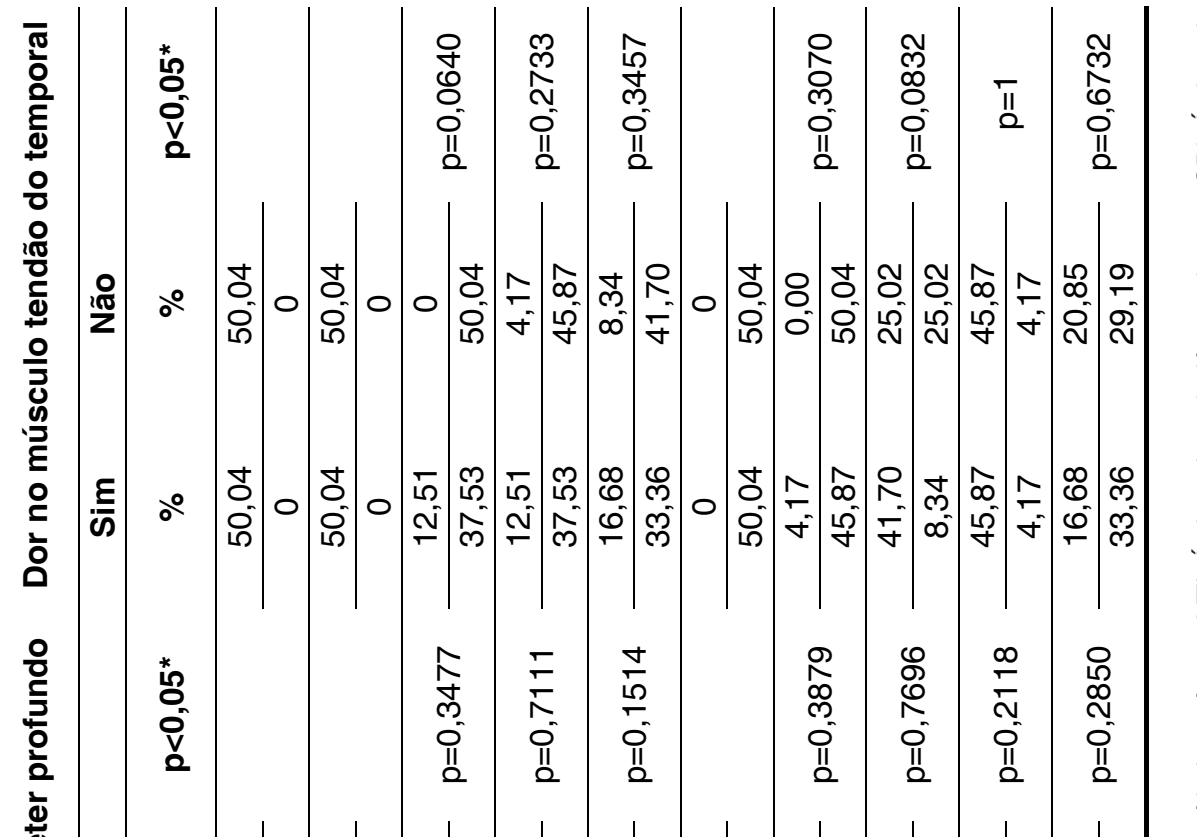

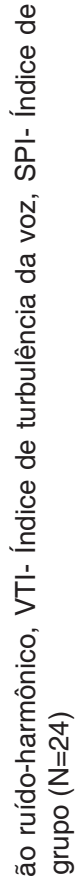

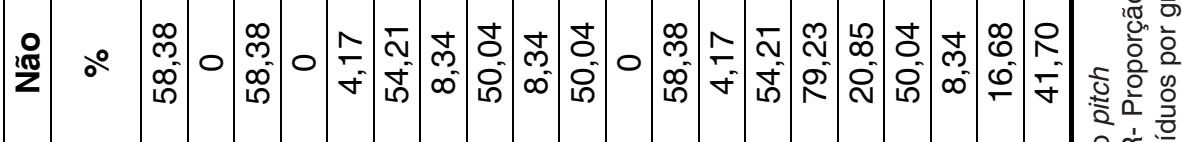

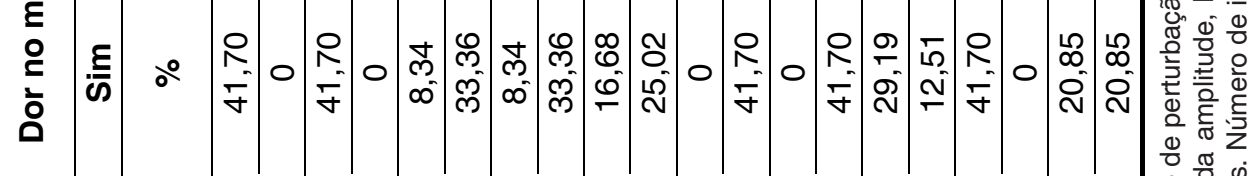

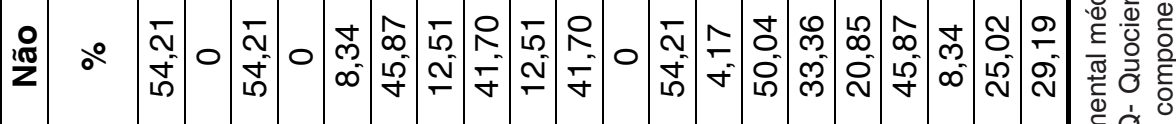

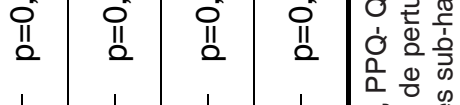

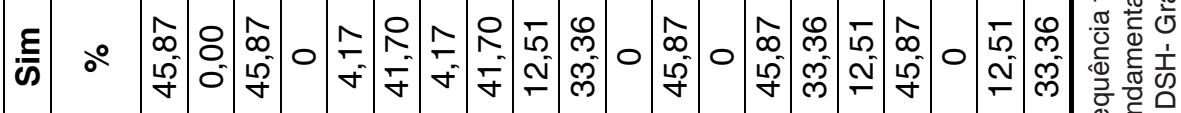

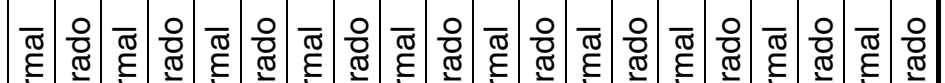




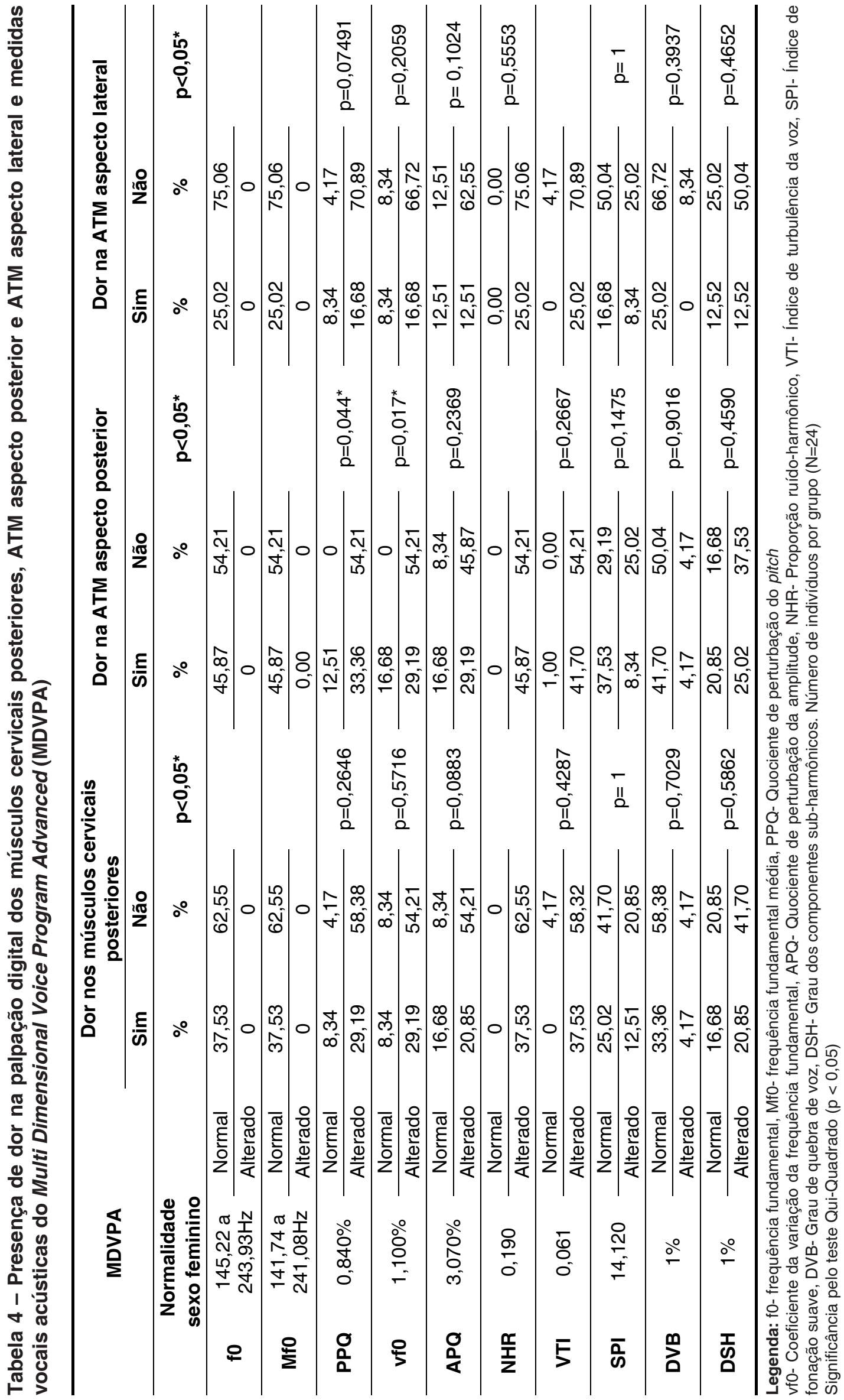




\section{DISCUSSÃO}

Este estudo procurou relacionar a presença de dor nos grupos musculares da cabeça, face, boca e pescoço envolvidos no processo fisiológico da função mastigatória e nos aspectos lateral e posterior da ATM com medidas do sinal acústico da voz em mulheres com sintomatologia de DTM. Para verificar a existência desta relação, no grupo estudado, foi realizado por meio de análise estatística, o cruzamento entre as medidas vocais acústicas de frequência fundamental (f0), frequência fundamental média (Mf0), Quociente de perturbação do pitch (PPQ), Coeficiente da variação da frequência fundamental (vf0), Quociente de perturbação da amplitude (APQ), Proporção ruído-harmônico (NHR), Índice de turbulência da voz (VTI), Índice de fonação suave (SPI), Grau de quebra de voz (DVB) e o grau dos componentes sub-harmônicos (DSH) com a presença de dor na musculatura intra e extraoral e nos aspectos lateral e posterior da ATM.

Neste trabalho verificou-se significante alteração das medidas de PPQ e vfO, na ausência de dor no aspecto posterior da ATM e alteração do VTI na ausência de dor no músculo masseter superficial (Tabelas 2 e 4), sugerindo que, mesmo na ausência de dor, sujeitos com sintomatologia da DTM podem apresentar alterações acústicas da voz.

O quociente de perturbação do pitch (PPQ) e o coeficiente da variação da frequência fundamental (vf0) são medidas de perturbação relacionadas à frequência vocal. Essas medidas traduzem a irregularidade da vibração da mucosa das pregas vocais (PPVV), o jitter, correlacionando-se com as características biomecânicas das PPVV e com a variação do controle neuromuscular. As alterações de jitter podem ser decorrentes do aumento da massa ou da tensão das pregas vocais, da simetria das estruturas ou, ainda, das funções muscular ou neural envolvidas ${ }^{17,18}$.

Por outro lado, o índice de turbulência da voz (VTI) apresenta maior relação com os componentes de turbulência aérea que correspondem a modificações na adução glótica ${ }^{17}$.

As DTM estão frequentemente associadas à hiperatividade muscular, mas também podem ocorrer na presença de músculos hipoativos, principalmente os músculos mastigatórios masseteres ${ }^{19}$. Esta afirmação vem ao encontro dos achados deste artigo em que, mesmo na ausência de dor desta musculatura elevadora da mandíbula, o parâmetro vocal acústico VTI, relacionado à adução das PPVV, pode sofrer mudanças durante a fonação desses sujeitos, também podendo influenciar 0 PPQ e a vf0, que se relacionam à perturbação da frequência de vibração das PPVV.
É possível sugerir esta relação ao se considerar que a produção da voz está diretamente relacionada a toda musculatura, que, de alguma forma, influencia a movimentação da laringe ou que, por desorganização na realização de suas funções, acaba sobrecarregando-a. Na literatura, refere-se que a musculatura avaliada no presente trabalho é a mesma que influencia a estabilização da mandíbula que, por sua vez, é co-participante na estabilização dos músculos que realizam a deglutição e a fonação ${ }^{20}$.

Estes achados sugerem que a DTM pode interferir nas medidas acústicas da fonte glótica por outras variáveis que não a dor muscular. Tal fato concorda com um estudo cujos achados mostraram que uma das variáveis da DTM, o grau de severidade, ocasiona diminuição da loudness, e alteração na ressonância da voz, interferindo na qualidade vocal $^{3}$.

Outra pesquisa, em que não foi encontrada relação entre as alterações das medidas do sinal acústico da fonte glótica e a presença de dor nos músculos avaliados, também suporta os achados do presente estudo (Tabelas 2 e 4). Os autores avaliaram as características do movimento mandibular em indivíduos com DTM e em assintomáticos e analisaram as possíveis interferências dessas disfunções e as implicações de severidade quanto ao índice de dor nesses indivíduos. Os resultados mostraram que a presença de DTM acarreta redução das amplitudes máximas de abertura e redução da velocidade tanto de abertura quanto de fechamento dos movimentos mandibulares e os diferentes graus de dor parecem não determinar maior redução desses valores ${ }^{21}$.

Porém, outro estudo afirma que a presença de dor em pessoas com DTM se reflete na movimentação da ATM, deixando-a com movimentos mais restritos, podendo influenciar os parâmetros de qualidade vocal ${ }^{22}$. Além disso, é importante mencionar estudos que relacionaram a amplitude do movimento da mandíbula com o pitch da voz e encontraram relação diretamente proporcional ${ }^{23,24}$.

Em pesquisa com indivíduos assintomáticos, verificou-se que durante a fala ocorrem discretos movimentos mandibulares anteroposteriores e laterais ${ }^{25}$. Entretanto, em indivíduos com DTM observou-se que é comum a redução desses movimentos durante a articulação da fala ${ }^{5}$. Também foi verificada dificuldade de abertura bucal em indivíduos com queixa de hipertrofia do músculo masseter e rigidez muscular ${ }^{26}$.

Outro estudo afirma que esta redução de movimentos pode ser relacionada à presença de dor ou desconforto na ATM, podendo, de forma análoga à limitação do movimento da mandíbula, ocorrer 
esforço laríngeo e alteração da qualidade vocal de sujeitos com $\mathrm{DTM}^{27}$. Na presente pesquisa, não foi possível confirmar esta relação entre a dor muscular e a alteração da voz (Tabelas 2 e 4).

No entanto, a DTM pode vir acompanhada de tensão muscular, com travamento na abertura da mandíbula, tensão excessiva na cintura escapular, e o pescoço e os ombros podem estar alterados em sua posição no espaço. Assim, a laringe pode estar também fixa no pescoço, como conseqüência dessa tensão e vir a alterar a qualidade da voz ${ }^{8}$.

Determinadas alterações articulatórias podem exigir um ajustamento muscular que envolva alteração no dinamismo da laringe, dificultando uma emissão equilibrada ${ }^{8}$.

\section{CONCLUSÃO}

Não houve relação entre as medidas de voz alteradas e a presença de dor nos grupos musculares da cabeça, face, boca e pescoço envolvidos no processo fisiológico da função mastigatória e nos aspectos lateral e posterior da ATM das mulheres com sintomatologia de DTM participantes deste estudo.

\begin{abstract}
Purpose: to check the relationship between pain presence in head, face, mouth and neck muscles involved in the physiological process of chewing function and in the lateral and posterior aspects of temporomandibular joint (ATM) and acoustical glottic source vocal measures. Method: 24 women with ages varying between 16 and 56 year old with signs and symptoms of Temporomandibular Joint Dysfunction (TMD) took part of this study. We applied an Anamnesis questionnaire, a specific clinic exam to check pain presence in head, face, mouth, neck muscles and in the ATM, otorhinolaryngology, stomatognatical and pure tone audiometric evaluations, the voice recorder and the acoustic voice analysis by the Multi Dimensional Voice Program Advanced software from KayPentax. The results were analyzed thought the qui-square test with statistical significance of $(p<0.05)$. Results: there were statistical significance between the pain absence in the superficial masseter and the alteration in the acoustic vocal parameter, such as Voice Turbulence Index (VTI); pain absence in the temporomandibular posterior aspect joint and the alteration of pitch perturbation quotient (PPQ) and the fundamental frequency variation (Vfo); pain presence in the medial pterygoid area and normality in the degree of voice breaks (DVB). Conclusion: we found that there was no statistical significance between the alteration in voice parameters and the presence of pain in the evaluated muscles, but some parameters with alteration can have a relation with pain absence; this suggests that other aspects of temporomandibular joint disorder can interfere in the acoustical voice parameters.
\end{abstract}

KEYWORDS: Temporomandibular Joint; Facial Pain; Voice Disorders

\section{REFERÊNCIAS}

1. Vazquez-Delgado E, Schmidt JE, Carlson CR, DeLeeuw R, Okeson JP. Psychological and sleep quality differences between chronic daily headache and temporomandibular disorders patients. Cephalalgia. 2004; 24(6):446-54.

2. Bianchini EMG. Mastigação e ATM: avaliação e terapia. In: Marchesan IQ. Fundamentos em fonoaudiologia: aspectos clínicos da motricidade oral. Rio de Janeiro: Guanabara Koogan; 1998. p. 37-49.

3. Silva AMT, Morisso MF, Cielo CA. Relação entre grau de severidade de disfunção temporomandibular e a voz. Pró-Fono. 2007; 19(3):279-88.
4. Oliveira MFR, Crivello JR. O comportamento da movimentação mandibular em pacientes com disfonia funcional e organofuncional. J Bras Fonoaudiol. 2004; 5(19):110-7.

5. Celic R, Jerolimov V, Zlataric DK. Relationship of slightly limited mandibular movements to temporomandibular disorders. Braz Dent J. 2004; 15(2):151-4.

6. Machado IM, Bianchini EMG, Silva MAA, Ferreira LP. Voz e disfunção temporomandibular em professor. Rev. CEFAC. 2009; 11(4):630- 43.

7. Santos PPA, Santos PRA, Souza LB. Características gerais da disfunção

temporomandibular: conceitos atuais. Rev Naval Odontol On Line. 2009; 3(1): 10-3. 
8. BEHLAU, M. S. Avaliação da voz. In: BEHALU, M. S. Voz: o livro do especialista I. Rio de Janeiro: Revinter, 2001. cap. 3, p. 91-172.

9. Santos ECA, Bertoz FA, Pignatta LMB, Arantes FM. Avaliação clínica de sinais e sintomas da disfunção temporomandibular em crianças. Dental Press Ortodon Ortop Facial. 2006; 11(2):29-34.

10. Oliveira AS, Dias EM, Contato RG, Berzin F. Prevalence study of signs and symptoms of temporomandibular disorder in Brazilian college students. Braz Oral Res. 2006; 20(1):3-7.

11. Merighi LBM, Silva MMA, Ferreira AT, Genaro KF, Berretin-Felix G. Ocorrência de disfunção temporomandibular (DTM) e sua relação com hábitos orais deletérios em crianças do município de Monte Negro - RO. Rev CEFAC. 2007; 9(4):497-503.

12. Stegenga $B$, Schouten $H$. Mandibular pain and movement disorders, focusing on the temporomandibular joint. Ned Tijdschr Tandheelkd. 2007; 114(1):41-6.

13. Tosato JP, Biasotto-Gonzalez DA, Gonzalez TO. Presence of temporomandibular joint discomfort related to pacifier use. Rev Bras Otorrinolaringol. 2005; 71(3):365-8.

14. Fonseca DM, Bonfante $G$, vale AL, Freitas FST. Diagnóstico pela anamnese da disfunção craniomandibular. Rev. Gaúcha Odontol. 1994; 42:23-8.

15. Okeson JP. Tratamento das desordens temporomandibulares e oclusão. São Paulo: Artes Médicas; 2000.

16. Angle EH. Malocclusion of the teeth. Philadelphia: SS White Dental Mfg Co; 1907.

17. Barros APB, Angelis EC. Avaliação perceptivoauditiva da voz. In: Dedivites RA, Barros APB. Método de avaliação e diagnóstico de laringe e voz. São Paulo: Lovise; 2002. p.201-21.
18. Cappellari VM, Cielo CA. Características vocais acústicas de crianças pré-escolares. Rev. Bras. Otorrinolaringol. 2008; 74(2):265-72.

19 Bérzin F. Surface eletromiography in the diagnosis of syndromes of the cranio-cervical pain. Braz J Oral Sci. 2004; 3(10):484- 91.

20. Rahal A, Lopasso FP. Eletromiografia dos músculos masseteres e supra-hióideos em mulheres com oclusão normal e com má oclusão Classe I de Angle durante a fase oral da deglutição. Rev. CEFAC. 2004; 6(4):370-5.

21. Bianchini EMG, Paiva G, Andrade CRF. Movimentos mandibulares na fala: interferência das disfunções temporomandibulares segundo índices de dor. Pró-Fono. 2007; 19(1):7-18.

22. Fischer MJ, Riedlinger K, Gutembrunner C, Bernateck M. Influence of temporomandibular joint on range of motion of the hip joint in patients with complex regional pain syndrome. J. Manipulative Physiol Ther. 2009; 32(5):364-71.

23. Lim M, Bones P. Vowel effect on glottal parameter and the magnitude of jaw opening. $\mathrm{J}$ Voice. 2006; 20(1):46-54.

24. Austin SF. Jaw opening in novice and experienced classically trained singers. $\mathrm{J}$ Voice. 2006; 21(1):72-9.

25. Bianchini EMG, Andrade CR. A model of mandibular movements during speech: normative pilot study for the Brazilian Portuguese language. Cranio. 2006; 24(3):197-206.

26. Mangilli LD, Rodrigues CS, Campiotto AR. A intervenção fonoaudiológica no pós-operatório da hipertrofia benigna do músculo masseter. Dent. Press Ortodon. Ortop. Fac. 2006; 11(2):103-9.

27. Taucci RA, Bianchini EMG. Verificação da interferência das disfunções temporomandibulares na articulação da fala: queixas e caracterização dos movimentos mandibulares. Rev Soc Bras Fonoaudiol. 2007; 12(4):274-80.
http://dx.doi.org/10.1590/S1516-18462011005000079

RECEBIDO EM: 15/06/2010

ACEITO EM: 06/04/2011

Endereço para correspondência:

Luane de Moraes Boton

Rua Dr. Astrogildo de Azevedo, no 328, Ap. 06

Centro, Santa Maria, RS

CEP: 97015-150

E-mail: luaneboton@yahoo.com.br 